\title{
El papel del entendimiento en la realidad humana
}

\author{
MARÍA DEL CARMEN PAREDES MARTÍN, \\ Universidad de Salamanca
}

En este trabajo iniciamos nuestra investigación sobre la importancia del entendimiento en la construcción de la filosofía de Hegel, como clave diferenciadora de su idealismo, por lo tanto, no sólo en la relación del entendimiento con la razón, sino por sí mismo. El aforismo de la época de Jena: "La razón sin entendimiento no es nada, mientras que el entendimiento sigue siendo algo incluso sin la razón. El entendimiento no se puede regalar" " parece responder a una posición fundamental de Hegel. Sin embargo, frecuentemente se piensa siempre en la razón hegeliana como algo independiente y en todo caso desvinculada del entendimiento. De hecho, este es un tema relativamente poco atendido en la bibliografía sobre Hegel, incluso en las publicaciones más recientes.

\section{Los "EscRitos DE JUVENTUD"}

Las referencias de Hegel al entendimiento, bien para describir una situación, establecer comparaciones, enjuiciar o criticar diversos aspectos de la filosofía o de la cultura se encuentran ya en sus escritos más tempranos. Por ejemplo, en el llamado "Fragmento de Tubinga" el entendimiento aparece vinculado a la Ilustración alemana y a algunas de sus obras, de esta manera:

"El entendimiento sirve sólo a la religión objetiva, para clarificar los principios y exponerlos en su pureza; ha producido frutos magníficos, como el Natán de Lessing y merece los elogios con los que siempre se le exalta.

Pero mediante el entendimiento los principios nunca llegan a hacerse prácticos.

El entendimiento es un cortesano que se orienta de buen grado según los caprichos de su señor; sabe cómo aducir fundamentos de justificación para cada pasión y para cada empresa -ante todo al servicio del amor propio, que siempre es muy perspicaz

1 G.W.F. Hegel, Werke in zwanzig Bänden, Theorie Werkausgabe (=TW), Suhrkamp, Frankfurt 1971, 2, 551; K. Rosenkranz, Hegels Leben, Berlin, 1844, reimpr. Darmstadt, WBG, $1963,546$. 
para dar un color bello a las faltas ya cometidas o por cometer - y a menudo se alaba a sí mismo por haber encontrado un pretexto tan bueno para sí.

La ilustración del entendimiento nos hace en verdad más prudentes, pero no mejores". 2

Observamos en este texto cómo vinculó el joven Hegel en 1793 la Ilustración, la religión objetiva y el entendimiento, expresando sus reservas sobre la bondad de las relaciones que se establecían entre ellos. Podríamos decir que esta asociación es doble; por una parte, se refiere a la que conecta la religión objetiva con el entendimiento y, por otra parte, a la que considera, de un modo más general, que la Ilustración (Aufklärung) es equiparable a "querer actuar por el entendimiento" (GW 1,94/158). En cuanto a la primera conexión, según Hegel, la religión objetiva interesa al entendimiento y la memoria, es decir, se refiere a conocimientos objetivados y reflexionados que pueden ser enseñados y aprendidos tal como se transmiten otros conocimientos objetivos. La religión subjetiva, en cambio, es la religión vivenciada en el sentimiento y plasmada en la acción, de tal manera que, "si digo de un hombre que tiene religión, esto no significa que tenga grandes conocimientos de la misma, sino que su corazón siente las acciones, los milagros, la proximidad de la divinidad, que su corazón reconoce y ve a Dios en su naturaleza, en los destinos de los hombres" (GW $1,88 / 152)$.

Acerca de la Ilustración, Hegel considera en este texto juvenil que su función principal ha consistido en formar al entendimiento respecto de los diversos objetos que interesan a la religión (cf. GW 1, 95,97/159, 161), así como a la filosofía teórica, a la filosofía práctica y a las restantes manifestaciones de la cultura, aunque no siempre esa tarea formativa ha redundado en un beneficio real para el sentido de la vida de los seres humanos. Los grandes avances del entendimiento ilustrado le merecen a Hegel este comentario: "cuanto más complejo es el edificio en el que trabaja toda la humanidad, menos le pertenece a cada individuo como propio." (GW 1, 99/162).

Estas primeras observaciones van adquiriendo una configuración más concreta en escritos posteriores. En los textos que Hegel escribió en Francfort aparece el tema de la oposición entre el entendimiento y sus formas de reflexión y la vida. Por ejemplo, en uno de los fragmentos más sugerentes de esa época Hegel considera la vida como un círculo en que se distinguen tres aspectos: 1) la unidad no desarrollada, 2) la formación (Bildung) y 3 ) la unidad completa. El

2 G. W. F. Hegel, "Religion ist eine der wichtigsten Angelegenheiten..." en: Frühe Schriften I, Gesammelte Werke (=GW) 1, ed. F. Nicolin y G. Schüler, Dusseldorf, 1989, 94. Traducción española de M ${ }^{a}$ del Carmen Paredes Martín, "El 'Fragmento de Tubinga"”, en: Revista de filosofía, $3^{\mathrm{a}}$ época, vol. VII (1994) núm. 11, Madrid, 158 (Se citará dentro del texto, con la paginación alemana seguida de la correspondiente a la traducción española). 
ciclo del desarrollo de la vida busca el retorno a la unidad, pero no a aquella de la que se parte, sino a una unidad plena y realizada que incorpora la reflexión. Sobre ello, escribe:

a partir de la unidad no desarrollada, la vida por medio de la formación ha recorrido el círculo hasta [alcanzar] una unidad completa; [...] en el desarrollo la reflexión produjo cada vez más contraposiciones..., hasta que el amor supera la reflexión en una ausencia total de objetividades"3.

No es casual que Hegel hable en este contexto del amor, pues en las líneas precedentes señala que el amor no es entendimiento, sino aquello en lo que el todo de la vida encuentra la resolución del desgarramiento de su unidad.

En un sentido semejante se expresa Hegel en el "Fragmento de Sistema", de 1800 , considerando que la reflexión introduce en la vida sus conceptos de relación y de separación, de lo individual, que subsiste por sí mismo, y lo universal, que se encuentra unido. En tales términos se habla de la vida fijada por la reflexión, pues toda expresión introduce en última instancia una posición unilateral. Sin embargo, Hegel hace notar asimismo que tampoco es acertado considerar la vida sólo como unificación y suponer que la oposición es introducida en ella desde fuera, o bien que la clave está en la unión de lo que la reflexión ha separado. Más concretamente, es necesario pensar a la vez la unión y su contrario, de modo que "la vida es la unión de la unión y de la no-unión". ${ }^{4}$ Con ello indica anticipadamente un tema que va a desarrollar en los próximos años: el de cómo pensar la unión de los opuestos desde una perspectiva no excluyente, cómo "pensar la vida pura" incorporando en ella la reflexión. ${ }^{5}$

\section{El ENTENDIMIENTO EN LA CRÍTICA DE Hegel A LAS CORRIENTES DE LA ÉPOCA.}

\section{II.1. LA DIFERENCIA}

Podemos decir, por tanto, que "determinar", “separar" y "abstraer" son términos que caracterizan al entendimiento en Hegel desde los textos de Francfort, características que se siguen poniendo de manifiesto en diferentes contextos hasta los escritos de madurez de Berlín. El entendimiento es una actividad del yo pensante y lo propio de él es "atenerse al punto de vista de lo finito como algo último". Esta caracterización general experimenta gradualmente ciertas modificaciones, mediante las cuales se hace mucho más compleja la función

3 "Die Liebe", en TW, 1, 246 (v. Nota 1).

4 Cf. TW 1, 422.

5 Sobre estos temas, cf. $M^{\text {a }}$ del Carmen Paredes-Martín, "La vie comme infinité concrète", en: Das Leben denken, Vol, II, 336-344, Akademie V., Berlin, 2007. 
y la crítica de Hegel, a la vez que el entendimiento adquiere progresivamente nuevos perfiles metodológicos. Además, la concepción hegeliana del papel del entendimiento marca diferencias importantes entre Hegel y Schelling, así como entre Hegel y los románticos. Pero en no menor medida supone también una reelaboración del entendimiento kantiano y a la vez una profunda ocupación con el planteamiento de la Crítica de la razón pura.

En la parte introductoria del escrito de la Diferencia tenemos el primer planteamiento de una orientación de Hegel a repensar la relación entre entendimiento y razón y de ese modo también modificar la visión de la subjetividad humana como un conjunto de compartimentos estancos que serían las facultades anímicas, aunque todavía el propio Hegel utiliza el término "facultad", que evitará en el futuro. En términos generales, encontramos un enjuiciamiento crítico del edificio del entendimiento, que se opone a la vida, que es considerada en esta época como el modo de ser de la realidad concreta. Pero esto no significa que la filosofía se vuelva ingenuamente contra la oposición y la limitación sin más, sino sólo contra la fijación absoluta de la escisión por parte del entendimiento.

La necesidad de la filosofía surge cuando el poder de unificación desaparece de la vida de los hombres y los opuestos pierden su viva relación e interacción y cobran autonomía. ${ }^{6}$

Precisamente, porque la escisión es necesariamente un factor de la vida, también es la fuente de la necesidad de la filosofía y la reflexión es un instrumento de la actividad filosófica.

Bajo este aspecto, el entendimiento de por sí se presenta como contrario a la vida y como una fuerza de limitación; por ello se hace necesario elaborar una nueva perspectiva dentro de la cual la capacidad limitadora del entendimiento encuentre su propia superación. Hegel plantea directamente el problema de la reflexión, dentro del cual el entendimiento es interpretado desde la relación entre reflexión finita, reflexión filosófica y conocimiento especulativo. Dicha relación se hace posible por el modo como operan el entendimiento y la razón, pues lo interesante no es tanto la distinción entre ambos, sino la relación que Hegel establece entre ellos a través de la reflexión. Justamente debido a esa relación, es decir, debido a que el entendimiento no es una facultad aislada, su propia reflexión puede llegar a ser reflexión filosófica y por lo tanto instrumento que abre el proceso hacia el pensamiento racional.

6 G.W.F. Hegel, Differenz des Fichteschen und Schellingschen Systems der Philosophie, GW 4,p. 14. Traducción española de $\mathrm{M}^{\mathrm{a}}$ del Carmen Paredes Martín, Diferencia entre el sistema de filosofía de Fichte y el de Schelling, Tecnos, Madrid, 1990, p. 20. (Se cita en adelante dentro del texto, con la paginación de GW seguida de la correspondiente a la traducción española). 
La relación entre el entendimiento y la razón se articula dinámicamente en torno a un proceso de aniquilación. Sin embargo, no se trata de que la razón aniquile al entendimiento, sino de que el entendimiento llegue a producir su aniquilación cuando, en el límite de sí mismo, vuelve sobre sí y conoce sus determinaciones como una serie de limitaciones negativas. Por consiguiente, la aniquilación del entendimiento tiene un significado metódico, en cuanto que gracias a ella la reflexión y sus formas de pensamiento llegan a ser una condición necesaria, aunque no suficiente, para el conocimiento filosófico especulativo. Por ello afirma Hegel:

Sólo en la medida en que la reflexión tiene referencia a lo absoluto es razón y su acto es un saber; pero mediante esta referencia desaparece su obra, sólo la referencia subsiste y es la única realidad del conocimiento; no hay por tanto ninguna verdad de la reflexión aislada, del pensar puro, sino la de su aniquilarse (GW 4, 19/30).

Pero también esa aniquilación tiene un contenido temático, ya que lo determinado por el entendimiento está limitado por lo indeterminado y esto, a su vez, no es nada para el entendimiento, o dicho de otro modo, sus propias determinaciones se muestran como negaciones. Este modo de considerar al entendimiento desde una perspectiva incipientemente especulativa proporciona la prueba lógica de su destrucción. El entendimiento se destruye de hecho cuando fija lo finito y lo infinito en contraposición recíproca, pues la oposición significa que, en cuanto uno de los términos es puesto, el otro es superado. La importancia de esta contraposición es central para Hegel, ya que a ella se dirige la tarea de la filosofía para pensar lo finito en lo infinito y tomar conciencia del significado y alcance de la misma. ${ }^{7}$ Ahora bien, el entendimiento está tan alejado de esta pretensión que desde la perspectiva intelectiva lo absoluto es propiamente "nada", es decir, lo indeterminable por medio de su reflexión. Esto parece indicar que en la Diferencia Hegel interpreta la reflexión como actividad de poner determinaciones opuestas del pensar, pero sin que esa reflexión pueda captar como suya la oposición que acompaña a esta actividad.

También la discusión con Fichte en el citado escrito tiene al entendimiento como una clave fundamental. En ella se puede destacar, sobre todo, cómo interpreta Hegel la posición de la Doctrina de la Ciencia contraponiendo la verdadera identidad especulativa a la identidad del entendimiento. Para Hegel, el planteamiento de Fichte responde a un verdadero pensamiento especulativo, si bien la identidad del principio "yo=yo" sería una identidad simple, que no logra exponer sistemáticamente la identidad absoluta, antes bien, se limita a reconstruir la contraposición entre sujeto y objeto. Otro punto interesante es la 
crítica a la oposición entre el yo y el mundo - en la discusión del tercer principio fichteano-, que se puede interpretar con un significado antropológico, o al menos ligado al desarrollo de la noción de espíritu subjetivo. Hegel critica la existencia de un antagonismo entre el yo y el mundo en estos términos: "Tan pronto como aparece el uno, el otro está aniquilado; pero puesto que este uno puede aparecer solamente bajo el predicado del contrario del otro y por ello con su concepto aparece el concepto del otro y a la vez lo aniquila, este uno mismo no puede aparecer" (GW 4, 39/66). Debido a esto permanece una defectuosa relación entre ambos términos, que Hegel califica de este modo: "El yo pone un mundo objetivo porque en la medida en que se pone a sí mismo se conoce como deficiente y con ello desaparece la absolutez de la conciencia pura. El mundo objetivo adquiere con respecto a la autoconciencia la relación por la cual él es una condición de ésta."( GW 4, 43/73). El predominio del punto de vista de la mera reflexión y del entendimiento discursivo hacen del yo un sujeto ideal aislado y de la naturaleza algo determinado y muerto. Con ello aparece una determinada forma del problema del dualismo, que Hegel mencionará sobre todo en escritos posteriores. ${ }^{8}$

\section{II.2. CREER Y SABER}

No menos importante es la discusión con Kant en Glauben und Wissen, (Creer y saber) de 1802. Esta discusión se anuncia muy brevemente en el Proemio de la Diferencia, especialmente con relación a las categorías kantianas: "En esta deducción de las formas del entendimiento está expresado con la mayor precisión el principio de la especulación, la identidad del sujeto y del objeto. Y esta teoría del entendimiento ha sido apadrinada por la razón.- Por el contrario, cuando Kant hace de esta identidad misma en cuanto razón un objeto de la reflexión filosófica, desaparece la identidad desde su propia base. Si el entendimiento antes había sido interpretado racionalmente, ahora, por el contrario, la razón es interpretada intelectivamente. Aquí se hace patente en qué grado subordinado se concibió la identidad del sujeto y del objeto." (GW $4,6 / 5)$. El problema por lo tanto se refiere, en parte, al modo en que Kant ha interpretado a la razón como entendimiento, y por ello no ha logrado un desarrollo auténticamente especulativo en la deducción de las categorías.

$\mathrm{El}$ análisis de Creer y saber, con respecto a Kant, incluye unas consideraciones lógicas acerca del juicio sintético a priori, que Hegel considera como una aportación muy significativa de Kant al idealismo, ya que ha podido ex-

8 Cf. Über das Wesen der philosophischen Kritik. (Sobre la esencia de la crítica filosófica): "todo aspecto de la naturaleza viviente, y por lo tanto también la filosofía, tiene que buscar medios de salvación frente a la filosofía cartesiana, que ha expresado en forma filosófica el dualismo acaparador de la cultura de la historia moderna en nuestro mundo nor-occidental..." GW 4, 126 . 
presar "la verdadera idea de la razón". ${ }^{9}$ Hegel escribe que en el juicio aparecen separados lo particular y lo universal (o el ser y el pensar) como sujeto y predicado. La síntesis a priori tendría entonces el significado de unir lo particular -en el lugar del sujeto- y lo universal - en el lugar del predicado- porque la posibilidad de la misma es la razón, en cuanto unidad originaria de estos elementos heterogéneos y opuestos. ${ }^{10}$ También señala Hegel que lo a priori en sentido racional y en cuanto concepto medio no se expresa en el juicio, sino en el silogismo (GW 4,328s.), pues el juicio no puede expresar en la cópula la unidad de lo particular y lo universal, sino enlazarlos como opuestos. Es decir, lo que sugiere esta interpretación es que en la noción kantiana de la síntesis a priori hay un planteamiento especulativo, aunque esta síntesis no puede quedar en la dimensión del entendimiento mismo, ya que la posibilidad a priori de la misma es anterior al juicio como tal.

Esta posición tiene su antecedente en textos de Francfort, donde Hegel consideró al juicio como división originaria, siguiendo de cerca a Hölderlin. Dicha interpretación venía a consistir en que el juicio no es ante todo un enlace lógico, sino una división ontológica, que luego se conecta, y de ahí la consideración de juicio (Urteil) como división originaria (Ur-Teilung). En este sentido, Hegel entendió entonces la estructura del juicio según el esquema de la separación o antinomia y la unificación. ${ }^{11}$ Cuando interpreta a Kant en Creer y Saber, parte del origen racional de la unidad entre lo universal y lo particular y de su cognoscibilidad. La posibilidad de los juicios sintéticos a priori es fundamentada por Hegel en esta idea de la razón, aunque no es el juicio, sino el silogismo la estructura que se adapta a la forma lógica racional (cf. GW 4, 329). Juicios sintéticos a priori significan por lo tanto, para Hegel, unificaciones de determinaciones desiguales, incluso contrapuestas, cuyo fundamento es la identidad absoluta. ${ }^{12}$ En la base de estos argumentos se encuentra la cuestión de cómo concebir lo a priori dentro de un planteamiento trascendental y sobre todo de su desarrollo ulterior.

9 Cf. Glauben und Wissen oder die Reflexionsphilosophie der Subjectivität, in der Vollständigkeit ihrer Formen, als Kantische, Jacobische und Fichtesche Philosophie (Creer y saber, o la filosofía de la reflexión de la subjetividad, en la completud de sus formas, como filosofía kantiana, jacobiana y fichteana), GW 4, 315-414, p. 326.

10 Así lo afirma Hegel también en el $\$ 40$ de la Enciclopedia.

11 Cf.TW 1, 250-254. "Glauben ist die Art...", diciembre 1797/comienzos 1798,(Schüler No. 72), Nohl, 382s/R243s. Sobre este escrito tuvo gran influencia el texto de Hölderlin, Über Urtheil und Seyn (Abril 1795).cf. D. Henrich, "Hölderlin über Urteil und Sein”, en: HölderlinJarhbuch 14, Tübingen 1965-6, pp. 73-96.

12 Cf. K. Düsing, Das Problem der Subjektivität in Hegels Logik. Systematische und entwicklungsgeschichtliche Untersuchungen zum Prinzip des Idealismus und zur Dialektik, Hegel-Studien, Beiheft 15, Bonn, 1995, pp. 111ss; también H. Marcuse, Ontología de Hegel y teoría de la historicidad, Barcelona 1976, pp. 30ss. señala estos aspectos. 
Como es sabido, no es la filosofía de Kant el único tema de discusión en Creer y saber. Jacobi, y de nuevo Fichte, forman asimismo parte de ella, abordados también desde la perspectiva de su condición de "filosofías de la reflexión de la subjetividad". Por supuesto, el hecho de que Hegel unificara en gran medida la orientación de su crítica bajo el denominador de "filosofías de la reflexión" supone que en ellas la reflexión estaba ligada a la capacidad disociadora del entendimiento. Aquí, su concepción del entendimiento es negativa, puesto que el saber de estas filosofías estaba limitado a lo finito y empírico precisamente por el uso que hacen de los conceptos del entendimiento, en tanto que no encuentran relación alguna con la razón.

La múltiple caracterización del entendimiento en Creer y saber, bajo el criterio de la reflexión, se puede resumir como sigue:

1. El verdadero problema del idealismo es el de haber establecido al entendimiento como instancia suprema de conocimiento y como facultad aislada y separada de la razón. Este problema se presenta bajo aspectos diferentes.

2. El entendimiento sólo produce una síntesis formal, y de ahí el reproche de formalismo que Hegel dirige a las filosofías de la reflexión.

3. El problema de lo sintético, para Hegel, se encuentra en una insuficiente concepción de lo a priori, debida a haber sido referida esta concepción al entendimiento, como unidad formal de fenómeno y categoría.

4. El entendimiento sólo puede alcanzar una identidad relativa $-\mathrm{y}$ no una verdadera identidad - puesto que se define frente a la sensibilidad empírica, que suministra la materia del conocimiento. Se da por tanto también en este caso una forma de dualismo entre sensibilidad y entendimiento que es preciso abandonar.

En particular, a partir de la identidad relativa del entendimiento se produce una deducción de las categorías que es contingente y superficial, como deducción de las condiciones extrínsecas de los juicios. Por estas razones, en las filosofías de la reflexión Hegel ve expresada, bajo modelos distintos, una absolutización del entendimiento, que cosifica tanto a la filosofía como al mundo que nos presenta.

Además de lo que se acaba de indicar, está el problema de la subjetividad: en esta su primera discusión sistemática con Kant, Jacobi y Fichte, Hegel establece entre ellos un paralelismo, que ha sido calificado de "sorprendente" ${ }^{13}$ bajo el título de "filosofías de la reflexión de la subjetividad". Es decir, Kant, Jacobi y Fichte representaban una exposición diferente del principio de la subjetividad, que se fundamentaba en la oposición reflexiva entre conocimiento y objeto, convirtiéndola en eje del contenido de la filosofía. Cada uno de ellos habría 
elaborado sistemáticamente esta oposición quedándose en el lado subjetivo de la misma, es decir, haciendo de la subjetividad el elemento determinante.

La discusión que Hegel lleva a cabo en Glauben und Wissen formaba parte de la tarea de situarse con respecto a la filosofía contemporánea y de determinar la propia posición, precisando a la vez su concepción de la filosofía. Lo peculiar de ese escrito es que no se trata de una recensión ni se refiere a un determinada publicación contemporánea, sino a contenidos filosóficos propiamente dichos y al papel que el entendimiento desempeña en ellos.

\section{LA INFLUENCIA DE SPINOZA}

En esta tarea de situarse críticamente en el contexto filosófico de la época así como en la formación de la filosofía hegeliana, Spinoza juega un papel muy importante según han destacado diversos estudios. ${ }^{14}$ A juicio de Maluschke, Spinoza es el filósofo no dialéctico que más ha influido en la dialéctica de Hegel ${ }^{15}$ y la sintonía entre ambos no es tanto por el método, sino más bien por el asunto principal y la intención especulativa que les caracteriza, pues en el fondo de la elaboración hegeliana del concepto hay una reflexión profunda sobre la sustancia spinoziana, que comienza bastante temprano.

No solamente se puede afirmar esto con relación al tema del concepto, sino que en general el reconocimiento del punto de vista spinoziano, especialmente la dimensión metafísica, es evidente en los textos de los primeros años de Jena. Además, la filosofía de Spinoza es para Hegel una referencia reiterada en la interpretación de otras filosofías. Por consiguiente, la influencia de Spinoza en Hegel se puede encontrar en los puntos siguientes:

1. En la crítica a las filosofías de la reflexión de Kant, Jacobi y Fichte, que se mencionó en el apartado anterior. La mencionada crítica está ligada a la construcción típicamente hegeliana que conlleva poner de manifiesto las limitaciones de otras filosofías. Ejemplos de la influencia de Spinoza son las observaciones sobre la teleología de la Crítica del Juicio, que para Hegel está basada en un malentendido de la unidad spinozista. Hegel se refiere a la observación que hace Kant en el §73 de dicha obra sobre la explicación de la finalidad, diciendo que "el spinozismo no cumple lo que quiere". Para Hegel, el idealismo de los fines que Kant critica no es sino resultado de ver en la unidad

14 Cf. p. ej., G. Maluschke, Kritik und absolute Methode,H-St. Beiheft 13, Bonn 1974, 62-75; H.-Ch. Lucas, Wirklichkeit und Methode in der Philosophie Hegels : Untersuchungen zur Logik Der Einfluss Spinozas, Köln, 1974; del mismo autor, "Spinoza, Hegel, Whitehead: Substance, Subject and Superject" en: G.R. Lucas Jr., (ed.) Hegel and Whitehead. Contemporary Perspectives on Systematic Philosophy, SUNY, Albany, 1986. 39-59; F. Michelini, Sostanza e assoluto. La funzione di Spinoza nella "Scienza della logica" di Hegel, Dehoniane, Bologna 2004.

15 G. Maluschke, o. c., 55. 
spinozista una unidad del entendimiento discursivo, abstracta y sin finalidad, en lugar de comprenderla como unidad inteligible y orgánica, como unidad de las partes en el todo e identidad de la causa y el efecto (cf. GW 4.320s).

2. Con respecto a Jacobi, Hegel afirma que la interpretación de Spinoza en sus Cartas es una fusión del empirismo de Locke y de Hume. ${ }^{16}$ También critica el supuesto de que Spinoza, según Jacobi, no comprende el principio de razón y considera al tiempo como algo en sí. Con respecto a este punto, Hegel explica ampliamente conceptos spinozistas, como el de infinitum actu (cf. GW 4, 340) y señala que esta noción de infinito "como afirmación absoluta de una naturaleza cualquiera" y de lo finito "como negación parcial" 17 es un concepto absoluto e indivisible, que comprende en sí lo finito. Por eso considera Hegel que la idea de lo infinito -sobre la cual él tiene que elaborar su propia posición - es una de las más importantes de la filosofía de Spinoza. Fichte es asimismo parcialmente equiparado a Jacobi frente a Spinoza, en la medida en que el principio del yo es sólo una derivación de la identidad absoluta, con respecto a la cual Spinoza ha sabido ver la profunda idea de la unidad de pensamiento y ser (cf. GW 4, 397ss).

3. La base de estos elementos tomados de Spinoza se encuentra en la Diferencia. Allí afirma Hegel, sobre el principio fundamental (Grundsatz) de la filosofía, que la primera proposición de Spinoza no es una proposición del entendimiento, sino una proposición de la razón, pues contiene la identidad de los opuestos y su unificación en una contradicción (cf. GW 4.24/39). También la caracterización de la filosofía como autoconocimiento de la razón está en cierta consonancia con la doctrina del "amor Dei intellectualis" 18 y desde luego el concepto de absoluto como totalidad subjetiva y objetiva o como "sistema de la inteligencia y de la naturaleza" se puede aproximar a una perspectiva spinoziana, según lo sugiere la cita de Hegel: "el orden y conexión de las ideas es el mismo que el orden y conexión de las cosas" (GW 4,71/127).

4. El concepto de sustancia tiene un peso notable en los primeros Esbozos de Jena, que es donde Hegel por vez primera elabora esta categoría, teniendo a Spinoza como trasfondo de lo que Hegel llama "relación de sustancialidad". Ésta sirve a Hegel de modelo para toda relación, con la particularidad de que expresa una relación del ser. Para exponer los momentos de la relación de sustancialidad, Hegel se sirve de las categorías de posibilidad, realidad y necesidad,

16 Cf. GW 4, 331. F. H. Jacobi, Über die Lehre des Spinoza, in Briefen an Herrn Moses Mendelssohnn (Sobre la doctrina de Spinoza, en cartas al Sr. Moses Mendelssohn), en: Werke, IV, 1,2. Alusión de Hegel al escrito de Jacobi, David Hume über den Glauben, oder Idealismus und Realismus. Ein Gespräch (David Hume sobre el creer, o idealismo y realismo. Un diálogo), en: Werke, II, hrsg. F. Roth y F. Köppen. Reprod. Darmstadt, WBG, 1980.

17 Cf. B. Spinoza, Ética, I, Prop. VIII, esc. 1.

18 cf. o. c., V., Prop. 36; GW 4, 21/33s. 
las cuales son modos de determinación de la relación de sustancialidad. Por eso, para determinar más precisamente la categoría de sustancia, Hegel utiliza las categorías kantianas de la modalidad — que según Kant no tienen una referencia objetiva - puesto que sólo exponen relaciones particulares del pensamiento. Dicho de otro modo, Hegel quiere transformar la sustancia spinoziana en algo que actúe subjetivamente - es decir, la sustancia también tiene que caracterizar a un sujeto, a algo vivo, activo y dotado de reflexión - , a la vez que va más allá de Kant, pues para Hegel las categorías de modalidad sí que expresan una conexión de lo subjetivo con lo objetivo.

5. No obstante, esta primera concepción de una metafísica de la sustancia va siendo desplazada en favor de una "metafísica de la subjetividad", como ya pone de manifiesto el Esbozo de Sistema de $1804 / 5 .{ }^{19}$ Con ello no desaparece la atención a la sustancia, pero ésta pasa a formar parte del desarrollo categorial de la "metafísica de la subjetividad", donde el conocimiento tiene un significado central. Hegel afirma que la idea del conocer es lo primero de la metafísica, puesto que ella nos proporcionará el acceso a un sistema de principios (Grundsätze) lógico-metafísicos para luego establecer los distintos modos de ser de una metafísica de la subjetividad. ${ }^{20}$ Eso no significa que desaparezca la presencia de Spinoza en el sistema de Hegel, como lo indican referencias muy significativas en la Ciencia de la Lógica y en la Enciclopedia ${ }^{21}$ pero sí que parece claro que Hegel comienza a establecer una distancia importante respecto a planteamientos anteriores al introducir en la sustancia la dimensión de la subjetividad.

En lo que respecta al tema que nos ocupa, entendemos que la recepción de Spinoza por parte de Hegel, ya desde la época de Francfort, le sirvió en particular para minimizar frente a otras posiciones filosóficas la primacía de la conciencia. En este sentido, puede considerarse que la discusión de Hegel con las filosofías de la reflexión tiene en su trasfondo la filosofía de Spinoza en una medida considerable, aunque no sea la única.

19 En GW 7, 138-142, 182 dice Hegel: "la sustancia es más bien sujeto - lo uno de la sustancialidad y de la subjetividad; y no verdaderamente sustancia ni verdaderamente sujeto".

20 Sobre este planteamiento de una "metafísica de la subjetividad", cf. H. Kimmerle, Das Problem der Abgeschlossenheit des Denkens. Hegels 'System der Philosophie' in den Jahren 1800-1804, Hegel-Studien, Beiheft 8, Bonn, 1970, 1982, 129ss.; K. Düsing, Das Problem der Subjektivität in Hegels Logik., o. c., 189ss., y del mismo autor, "Von der Substanzmetaphysik zur Philosophie der Subjektivität", en: H. Kimmerle (ed.), Die Eigenbedeutung der Jenaer Systemkonzeptionen Hegels, Akademie V. Berlin 2004, 185-199.

21 Cf. por ejemplo, Ciencia de la Lógica, Libro I, cap. 2; Enciclopedia \$50 Nota. Sobre las diferencias entre determinados planteamientos de Hegel y Spinoza, cf. el estudio de M. Álvarez Gómez en este volumen, "La personalidad como vida en la verdad", así como en su obra, Experiencia y sistema. Introducción al pensamiento de Hegel, Salamanca, 1978, cap. II. 
No es la única, porque además de los contenidos filosóficos propiamente dichos en Creer y saber hay una consideración histórica del principio fundamental reflexivo de la Ilustración que para Hegel se ha consolidado y completado en Kant, Jacobi y Fichte. En la Introducción del citado escrito Hegel se refiere a la interpretación del mundo en la modernidad según el primado del entendimiento y sus conceptos que estableció en primer lugar Locke, en su Ensayo sobre el entendimiento humano (cf. GW 4,315s) y luego desarrolla la discusión con las "filosofías de la reflexión" de Kant, Jacobi y Fichte. Desde luego, la concepción del entendimiento en Spinoza queda a salvo de esta crítica.

\section{EL ENTENDIMIENTO ESCÉPTICO}

Un problema añadido a los que se han señalado en relación con las filosofías de la reflexión es el del escepticismo. Esto tenía que ver con el concepto de filosofía y su fundamentación y con las propuestas que se hacían para complementar y ampliar el planteamiento trascendental, en concreto en cuanto a los límites del conocimiento. También el tema de la verdad era un problema en el momento histórico de Hegel, debido al supuesto de su carácter inaccesible para la filosofía. A todo este debate se vinculaba la distinción kantiana entre método escéptico y escepticismo propiamente dicho, lo que contribuyó al desarrollo de posiciones escépticas. ${ }^{22}$

Hegel se ocupa de la cuestión de si era posible una refutación efectiva de la posición escéptica prácticamente en todos los ensayos de esa época hasta el escrito sobre el Derecho Natural (1803), porque en ellos el problema del escepticismo está presente de una manera u otra. Su crítica se dirige al escepticismo más reciente, es decir, al que proliferó en la filosofía postkantiana. Aunque no vamos a referirnos detalladamente a este tema,${ }^{23}$ conviene mencionar que además de criticar al escepticismo contemporáneo en Relación del escepticismo con la filosofía, considera Hegel que el verdadero escepticismo no constituye una cosa particular de un sistema, sino que él mismo es el lado negativo del conocimiento de lo absoluto y presupone inmediatamente a la razón como el

22 I. Kant, Crítica de la razón pura, A424: en la Dialéctica trascendental, Antitética de la razón pura (antes de tratar los conflictos de las ideas trascendentales) Kant afirma que el método escéptico es completamente distinto del escepticismo. Éste constituye un principio de ignorancia metódica que socava las bases del conocimiento, aquel consiste en observar la lucha entre dos contendientes para que diriman sus litigios, prescindiendo de si la causa por la que luchan es buena o mala. La razón se debería guiar por este método para sacar sus propias enseñanzas de la lucha misma.

23 Cf. $\mathrm{M}^{\mathrm{a}}$ del Carmen Paredes - Martín, "Introducción" a la traducción española del escrito de Hegel, Verhältnis des Skepticismus zur Philosophie, Darstellung seiner verschiedenen Modificationen, und Vergleichung des neuesten mit dem alten, GW 4, 197-238. (Relación del escepticismo con la filosofía, Biblioteca Nueva, Madrid, 2006). En lo sucesivo se cita esta traducción a continuación de GW 4. 
lado positivo (GW 4, 207/65).

Ejemplo de esta manera de entenderlo es el Parménides de Platón, que para Hegel es un exponente de la verdadera filosofía y al que califica de auténtico escepticismo, porque "abarca y destruye todo el ámbito de ese saber por conceptos del entendimiento" (l. c.). Por consiguiente, el escepticismo también se muestra aquí vinculado con el uso del entendimiento y la autenticidad del escepticismo platónico reside en que no duda de las verdades del entendimiento, sino en que niega completamente toda verdad del conocimiento que se pueda considerar de esa clase. Por eso, este escepticismo no es un aspecto particular de un sistema, sino el lado negativo del conocimiento filosófico; por eso también aunque en toda auténtica filosofía pueda encontrarse implícitamente un cierto escepticismo, en el Parménides está su figura explícita.

El escrito de Hegel sobre el escepticismo suscitó una amplia controversia, que en relación con este tema representa una etapa significativa en la historia previa a la Fenomenología. Sin duda la Introducción de esta obra tiene que ver con esa discusión sobre el proceder escéptico y la misma expresión hegeliana de que el método de la Fenomenología es un "escepticismo que se consuma a sí mismo" enlazaría con ella y consiguientemente iría también contra la posiciones de sus contemporáneos. Es significativo que años más tarde, en el Prólogo a la Fenomenología, Hegel se refiere al Parménides como "la más grande obra de arte de la dialéctica antigua". ${ }^{24}$

Por lo que se refiere a la posición de Hegel hacia el escepticismo en Jena, destacaremos aquí solamente lo siguiente:

a. Por una parte, Hegel establece una nueva relación entre skepsis y filosofía a partir de la cual elabora por vez primera el significado del proceder escéptico para la filosofía especulativa. A este procedimiento no lo llama aún "dialéctica", sino que lo pone en relación con el problema de las antinomias.

b. La inclusión del la skepsis no es solamente un asunto teórico, como se suele indicar, sino también práctico, ya que Hegel entiende que el escepticismo pirrónico abarcaba tanto la "subjetividad del carácter" como la "subjetividad del saber" (cf. GW 4, 221/80).

c. También Hegel aduce a Spinoza como ejemplo de auténtico escepticismo moderno, pues en el concepto de causa sui entra el principio del panti logoi logos isos antikeitai con toda su fuerza (cf. GW 4, 208/66), como una superación del principio formal de contradicción. Es decir, de la mano del spinozismo Hegel

24 GW 9.48. Sobre el escepticismo como método en la Fenomenología del espíritu, cf. M C. Paredes, "Escepticismo y dialéctica", en: Still Reading Hegel. 200 Years after the Phenomenology of Spirit, Universidade de Coimbra/ FCT Fundaçao para a Ciência e a Tecnologia, Coimbra, 2009,pp. 199-211. 
busca la integración del escepticismo en la filosofía mediante la radicalización de las antinomias. Por eso, la unidad de escepticismo y spinozismo y el uso sistemático de las antinomias prefiguran el método típicamente hegeliano en sus primeras elaboraciones. ${ }^{25}$

d. Al problema del escepticismo se unía la interpretación -principalmente sostenida por Jacobi - de que el idealismo trascendental disolvía toda objetividad en la nada, con lo que el conocimiento tendría únicamente una validez subjetiva. Hegel no puede admitir esta conclusión, que en el fondo traduce la cuestión de si el conocimiento puede trascender los límites de lo fenoménico en una disolución de la objetividad en las estructuras de la subjetividad humana. Por el contrario, la tesis kantiana de que no podemos conocer realmente las cosas en sí mismas se puede interpretar según Hegel en el sentido de que no hay "verdad" en lo fenoménico, pero las derivaciones de dicha tesis, que reducen el conocimiento a "los hechos de conciencia" desvirtúan el significado del auténtico escepticismo. Por lo tanto Hegel no piensa, como Jacobi, que el idealismo puede conducir a la disolución de la realidad del mundo objetivo; sino que hay un nihilismo del entendimiento, que no es el nihilismo de la nada absoluta. En cambio uno de los problemas del idealismo es pensar la nada absoluta, que está en el comienzo de la filosofía ${ }^{26}$ como vacío del pensamiento. ${ }^{27}$ O dicho de otro modo, ese nihilismo sería la intelección de la nada implicada en la contradicción inherente al lado negativo de la especulación.

Por consiguiente, Hegel complementa el concepto de escepticismo con el de nihilismo y, más allá de los términos de la polémica, comienza a tener en cuenta el problema de la negatividad. Es decir, el papel del escepticismo está vinculado en un primer momento para Hegel a un procedimiento de destrucción que tiene que aplicarse a los resultados que produce la conceptualización del entendimiento. ${ }^{28}$

\section{INTUICIÓN Y REFLEXIÓN}

Como se puede deducir de lo anterior, Hegel no sólo asume una concepción

25 Cf. M. Baum, Die Entstehung der Hegelschen Dialektik, Bonn 1989, 182ss.

26 GW 4, 399.

27 M. Alvarez, "El comienzo de la filosofía como vacío" en: $\mathrm{M}^{\mathrm{a}}$ C. Paredes (ed.), Intencionalidad, mundo y sentido. Problemas de Fenomenología y Metafísica, Salamanca, 2003, 13-38.

28 Otras afirmaciones posteriores dan idea del interés que sigue teniendo este tema. Por ejemplo, en la Enciclopedia leemos que "el escepticismo, en cuanto ciencia negativa llevada a término a través de todas las formas del conocer, podría ofrecerse como introducción (a la lógica) en la cual se pondría en evidencia la nulidad de tales proposiciones". Cf. G. W. F. Hegel, Enciclopedia de las ciencias filosóficas, traducción española de Ramón Valls, Alianza, Madrid, $2000 \S 78$, p. 182. 
del entendimiento que se basaba en Kant, al menos en parte, sino que también dependía de la interpretación que Hegel y otros hacían de la filosofía crítica. Por eso también la reflexión significaba frecuentemente - sobre todo en la época de Jena - , un modo de conocer unilateral, propio del entendimiento, que producía una multiplicidad de disociaciones. Concretamente, en esto consiste la reflexión propia del entendimiento, en producir conceptos cuyo contenido se delimitaba por contraposición a otro concepto entendido como contrario. Pero a la vez que Hegel maneja este concepto de reflexión, ligado a la lógica del entendimiento finito, va elaborando su propia concepción, que se apoya en la tesis de que la reflexión es un instrumento indispensable del pensar filosófico. Ello supone que se han de establecer el tipo de relación que guarda la reflexión y la intuición en el conocimiento especulativo.

En un principio, Hegel consideró compatible la reflexión con la intuición intelectual - que toma de Schelling - como la forma de conocimiento que podía superar los opuestos producidos por la reflexión. Esta intuición intelectual era un elemento necesario para el lado positivo de la especulación (cf. GW 4, 27/45). ${ }^{29}$ Sin embargo, Hegel comienza a abandonar esta posición bastante pronto y en Glauben und Wissen ya hay una crítica a la "intuición intelectual" de Fichte, en relación con el problema del formalismo. Pues no basta con que el yo posea la intuición intelectual de sí mismo para garantizar que con ella el idealismo queda a salvo de convertirse en un saber formal, según hace ver Hegel acerca del formalismo (cf. GW 4, 389s).

Este rápido desplazamiento se puede explicar en relación con algunos temas típicamente hegelianos. 1) Para Hegel en el seno de lo absoluto no hay disolución de la diferencia, sino lo contrario, la diferencia se mantiene de tal manera que hablar de lo absoluto es pensar la identidad y la diferencia conjuntamente. 2) Asimismo la exigencia de que lo absoluto sea cognoscible le lleva a reelaborar el concepto de reflexión de manera que pueda prescindir de la intuición intelectual.

En las lecciones de Lógica y Metafísica que impartió en el semestre de $1801 / 2$, las cuales se conocen por tres fragmentos ${ }^{30}$ y por apuntes de clase, ${ }^{31}$

29 En la Diferencia, la intuición trascendental se cualifica como la "identidad de lo subjetivo y lo objetivo en la conciencia", (GW 4,27s/44), y por eso afirma Hegel que en la intuición trascendental está superada toda oposición (cf. GW4, 27-28/44-47).

30 "Diese Vorlesungen..." GW5, 259; "Die Idee des absoluten Wesens", 262; Logica et Metaphysica: "Dass die Philosophie..."269ss. Estos fragmentos fueron publicados conjuntamente por E. Ziesche, «Unbekannte Manuskripte aus der Jenaer und Nürnberger Zeit im Berliner Hegel-Nachlass », en : Zeitschrift für philosophische Forschung, 29 (1975), 430-444. Rosenkranz publicó una parte de los mismos, cf. Hegels Leben, 189ss.; cf. GW5, 652s.

31 Se trata de apuntes recogidos por I.P.V. Troxler y editados por K. Düsing en: Schellings und Hegels erste absolute Metaphysik (1801-1802), Dinter, Köln, 1988. 
Hegel sitúa al entendimiento en el terreno de la lógica. ${ }^{32}$ Allí Hegel establece la oposición primaria de formas de conocimiento, no entre reflexión y especulación, sino entre intuición y reflexión. Por una parte, la construcción de la identidad absoluta para la conciencia es la tarea de la reflexión, lo que significa que la reflexión no consiste simplemente en quedarse en los opuestos, sino que es "el movimiento del pensar, para establecer la determinación y para superar la escisión en la unidad de lo absoluto. Permanecer en la contraposición describe sólo el carácter formal de la reflexión, que quiere captar lo absoluto en la forma de enunciados". ${ }^{33}$ Esta sería una "mala reflexión", pero la reflexión filosófica es la que va de uno a otro opuesto reasumiéndolos y aniquilándolos absolutamente.

Ahora bien, la lógica no parte de la reflexión, sino que es preciso contar con una intuición que, como condición del filosofar, haga posible el desarrollo de la lógica. Así que, por un lado, la intuición es en su conjunto la condición de la lógica y, por otro lado, entendiendo la reflexión como construcción de la idea de lo absoluto, Hegel busca la manera de elaborar especulativamente el concepto de reflexión, es decir, de reelaborar la reflexión filosófica.

En las lecciones posteriores Hegel comienza a desligar el concepto de reflexión tanto del de autoconciencia como del de entendimiento, según los había recibido de la filosofía de su tiempo y él mismo asumió. Esto lo lleva a cabo en el II Esbozo de Sistema de 1804/5, donde Hegel introduce (en la primera sección de la Lógica) un apartado (cuarto y último) sobre la infinitud. El concepto de infinitud, como concepto que abarca lo infinito y lo finito hace posible enunciar una teoría de lo absoluto y su manifestación, sin necesidad de una intuición previa. ${ }^{34}$ Es decir, lo absoluto en cuanto infinito que contiene lo finito, o como una contradicción absoluta, es una unidad de opuestos que no necesita ser captada intuitivamente.

Lo que esto viene a significar es que Hegel deja de considerar a la intuición como fuente independiente de conocimiento especulativo y como fundamento epistémico de la metafísica en una época inmediatamente anterior a la Fenomenología, con lo que la intuición pierde su preeminencia respecto de la reflexión y el concepto, si bien Hegel vuelve a pensar sobre las posibilidades de esta fuente

32 Por razones de espacio y de la densidad de este tema, sólo nos referimos a los puntos más destacados de este asunto.

33 W. Jaeschke, “Äusserliche Reflexion und immanente Reflexion”, en Hegel-Studien 13(1979), 85-117, 101.

34 GW 7. Jenaer Systementwürfe II. También en GuW se había referido Hegel a lo verdaderamente infinito, como la identidad de lo infinito y lo finito (GW 4,359). El concepto de infinitud, como concepto que abarca lo infinito y lo finito hace posible enunciar una teoría de lo absoluto y su manifestación, así como entender la lógica sin separarla de la metafísica (cf. M. Baum, Die Entstehung der Hegelschen Dialektik, Bouvier, Bonn, 1989, 226). 
de conocimiento en distintos momentos de la elaboración de su sistema.

Con todo, el papel del entendimiento se perfila insustituible en la filosofía de Hegel, no sólo por las reiteradas llamadas de atención a la irrupción y al predominio del mismo en la vida humana, sino también por la presencia del entendimiento en la filosofía y por los desarrollos que de sus diferentes usos se pueden derivar. 
